\title{
Performance des marchés de Défense : le rôle combiné des institutions et des choix contractuels
}

Thierry Kirat et Jean-Michel Oudot

\section{(2) OpenEdition \\ 12 Journals}

Édition électronique

URL : http://journals.openedition.org/ei/257

DOI : $10.4000 /$ ei.257

ISSN : 2553-1891

Éditeur

Association Économie et Institutions

Édition imprimée

Date de publication : 30 octobre 2009

Pagination : 93-111

ISSN : 1775-2329

\section{Référence électronique}

Thierry Kirat et Jean-Michel Oudot, «Performance des marchés de Défense : le rôle combiné des institutions et des choix contractuels », Économie et institutions [En ligne], 12-13 | 2009, mis en ligne le 31 janvier 2013, consulté le 19 avril 2019. URL : http://journals.openedition.org/ei/257 ; DOI : 10.4000/ ei. 257 


\title{
Performance des marchés de défense : (le rôle combiné des institutions et des choix contractuels
}

\author{
Thierry Kirat ${ }^{1}$ et Jean-Michel Oudot ${ }^{2}$
}

Les conditions de la coordination des organisations ont fait l'objet de nombreux développements tant dans la littérature juridique, économique que managériale. Les choix contractuels et institutionnels $\mathrm{y}$ sont présentés comme étant deux facteurs essentiels de la coordination des parties dans le cadre de relations entre organisations.

Nous proposons ici une illustration des rôles respectifs des institutions et des contrats d'une part et de leurs interactions d'autre part dans la coordination des parties à travers l'analyse du cas des marchés publics de défense en France. Ceux-ci constituant le premier poste d'achat et d'investissement du budget national, ils revêtent en effet une importance considérable. Nous cherchons plus précisément ici à expliquer les conditions d'exécution des marchés et leurs performances à travers l'étude de l'impact combiné des institutions, comprises comme étant l'ensemble des règles multilatérales formelles et informelles s'imposant aux parties dès lors qu'elles souhaitent interagir dans le cadre d'un secteur d'activité donné, et des choix contractuels, c'est-à-dire des décisions bilatérales prises spécifiquement par les parties en matière de passation d'un contrat, de rédaction des clauses du marché et leurs modifications ex post en vue de gérer l'élaboration et l'exécution d'une transaction. A la suite d'études centrées sur l'analyse des marchés de défense en France dans une perspective institutionnelle d'une part (Kirat, Bayon et Blanc 2003) et contractuelle d'autre part (Oudot 2007), il apparaît en effet utile de combiner ces deux approches de façon à rendre pleinement compte de leurs pouvoirs explicatifs combinés.

Nous empruntons pour ce faire à l'analyse économique du droit et à la théorie des coûts de transaction, deux cadres d'analyse particulièrement adaptés à la prise en compte des aspects de régulation en général et de coordination en particulier des relations entre organisations. Cette analyse théorique est complétée par une

1 CNRS-Institut de Recherche Interdisciplinaire en Sciences Sociales (Paris Dauphine)

2 Direction des affaires financières du ministère de la défense 
étude détaillée des conditions de mise en œuvre de 48 contrats signés par la DGA et 18 industriels français et européens entre 1994 et 2005. Notre contribution à la littérature existante se situe ici principalement sur le plan empirique à travers l'approfondissement de la connaissance des déterminants des conditions d'exécution et de la performance des marchés publics de la défense en France.

Deux aspects principaux et interdépendants de ces marchés sont étudiés de façon successive. Il s'agit en premier lieu des interdépendances de facto des marchés à la fois du côté de l'offre et de la demande. Etant donné que la réglementation prévoit que ces marchés soient indépendants les uns des autres, l'analyse des déterminants et des conséquences de leurs interactions se révèle particulièrement utile à la compréhension des pratiques actuelles. L'étude des renégociations de marchés à prix initialement fixe (c'està-dire forfaitaire) s'avère en second lieu également instructive de par ses déterminants et conséquences à la fois contractuels et institutionnels. Pour chacun d'eux, nous détaillons l'impact relatif des institutions d'une part et des choix contractuels d'autre part, tout en cherchant à mettre en avant leurs effets combinés sur la performance des contrats. Avant de développer ces aspects, nous présentons une brève synthèse du rôle des institutions et des contrats dans la coordination des parties.

\section{Section 1 - Institutions et contrats}

Sans prétendre opérer une recension exhaustive des définitions des institutions dans la littérature économique, nous nous en tenons à celles qui font autorité en la matière, sur la base de la Nouvelle économie institutionnelle (North, Williamson) : les institutions sont les règles du jeu dans lequel les organisations et les agents individuels se situent.

Deux problématiques centrales, complémentaires et interdépendantes, sont étudiées dans la littérature. La première a trait au niveau de coordination (Williamson, 2000 ; Brousseau et Raynaud, 2007 ; Pfister et al. 2006). Il s'agit dans cette perspective d'étudier quels sont les facteurs, institutionnels et/ou contractuels, qui sont les plus à même de coordonner les parties. Les interactions de ces niveaux de coordinations s'inscrivent dans ce champ d'étude. Les analyses qui font du contrat un phénomène simultanément contractuel et institutionnel, considérant que les règles du jeu sont moins l'environnement externe du contrat que constitutif des repères de la coordination contractuelle (Bazzoli et Kirat, 2003) appartiennent à ce champ. 
La seconde problématique principale porte sur les modalités de cette coordination. Que ce soit au niveau institutionnel ou contractuel, il est apparu dans cette perspective qu'à la fois des facteurs formels et informels participent à cet objectif. Alors qu'aux règles formelles sont associées le droit positif en vigueur, les règles informelles font référence aux mécanismes sociaux de l'exécution des obligations contractuelles, via les normes sociales, les principes déontologiques partagés dans une communauté sociale ou économique. Le caractère substituable et/ou complémentaire de ces facteurs de coordination a fait l'objet de nombreux développements dont une synthèse peut par exemple être trouvée dans Beuve et Saussier (2008). Un autre champ d'étude, en partie lié au précédent et qui nous intéresse ici, concerne les conditions d'exécution des arrangements, qu'ils soient formels ou informels (Baker, Gibbons et Murphy, 2002). Il a été souligné dans cette perspective que la confiance relationnelle serait d'autant plus grande que le cadre légal des transactions contractuelles est formalisé et susceptible d'être activé devant les tribunaux (Cf Deakin et Wilkinson, 2000).

Une question centrale dans ces deux problématiques est celle de l'exécution : les règles formelles sont-elles aussi peu importantes que les règles informelles dans cette perspective ? Plus précisément, quel est leur rôle relatif dans l'exécution des conditions initialement prévues des marchés? Cette notion d'exécution est couramment prise sous un angle assez large, comme un mécanisme qui oblige les parties à respecter leurs engagements initiaux. On doit à cet égard à Arrun $\square$ ada (2001) d'avoir séparé les mécanismes d'exécution en deux catégories, internes et externes aux parties, sur la base de l'idée que les institutions sont l'environnement du contrat sous l'angle des facteurs qui interviennent dans l'exécution : les coutumes, les normes sociales, éthique, ou autre, qui servent de références en vue de remplir ex post des contrats initiaux incomplets.

Les solutions individuelles à la coordination complexe sont soit les sanctions unilatérales basées sur une autorité, soit la non répétition des transactions (Baker, Gibbons et Murphy, 2002) et la prise d'otages (Williamson, 1985, 1996). Les solutions institutionnelles sont de leur côté associées à des solutions externes aux parties, car basées sur des normes et des règles non contractuelles : soit les usages et la coutume, à la base d'une exécution fait d'ostracisme ou de perte de réputation, soit le droit positif, associé à la saisine des tribunaux et d'une exécution basée sur la contrainte étatique ce qui présente certains coûts (Chakravarty et MacLeod, 2004). 
Les développements qui suivent s'inscrivent dans la lignée des travaux qui soutiennent que les contrats ne sont pas des isolats par rapport à l'environnement institutionnel. Ils s'en différencient toutefois par deux aspects : a) par l'idée que l'insertion institutionnelle des contrats n'est pas neutre, dans les modalités de la contractualisation et de la vie des contrats b) que toutes les institutions pertinentes ne se limitent pas à celle de l'exécution stricto sensu (notamment celle qui consiste en l'action des tribunaux dans le respect des engagements contractuels), compte tenu qu'il est impossible de faire l'impasse sur les catégories et les structures comptables, ainsi que sur les conditions du choix public et la dimension politique.

\section{Section 2 - Les interdépendances de facto des marchés}

Un facteur explicatif majeur de la performance des marchés d'armement est la complexité du processus de réalisation d'une part et du transfert de droits d'usage d'autre part. Le tableau suivant récapitule les risques les plus critiques dans l'approvisionnement de défense en France par ordre d'importance (Oudot, 2008a) :

\begin{tabular}{cl} 
Catégories de risques & \multicolumn{1}{c}{ Les déterminants critiques des catégories de risques } \\
\hline \multirow{2}{*}{ Risque contractuel } & $\begin{array}{l}\text { 1. Défaillance des entrées étatiques (en retard principalement) } \\
\text { 2. Evolution du besoin exprimé par l'Etat Major après la signature du contrat } \\
\text { 3. Difficultés liées à la non indépendance de plusieurs contrats }\end{array}$ \\
\hline \multirow{2}{*}{ Risque technologique } & $\begin{array}{l}\text { 4. Sous-estimation de la complexité de l'équipement faisant l'objet du contrat } \\
\text { 5. Complexité de l'équipement faisant l'objet du contrat } \\
\text { 6. Compétences techniques insuffisantes de la sous-traitance }\end{array}$ \\
\hline \multirow{2}{*}{ Risque industriel } & $\begin{array}{l}\text { 7. Compétences organisationnelles insuffisantes du titulaire } \\
\text { 8. Compétences organisationnelles insuffisantes de la sous-traitance }\end{array}$ \\
\hline \multirow{2}{*}{ Risque financier direct } & 9. Réduction des ressources suite à une décision de l'Etat Major \\
& 10. Blocage des ressources suite à une décision de l'Etat Major ou de la DGA \\
\hline
\end{tabular}

Tableau 1 : Les risques dans l'approvisionnement de défense en France

Il apparaît qu'à la complexité technique des équipements de défense (risque $n^{\circ} 5$ ) s'ajoute la complexité organisationnelle qui est susceptible d'expliquer bon nombre de risques intervenus dans le processus d'approvisionnement $\left(\mathrm{n}^{\circ} 1,2,3\right.$ et 4 en particulier), en association avec la rationalité limitée des contractants. Le recours à une technologie à la pointe de l'état de l'art appelle en particulier à l'interaction d'acteurs spécialisés dans les différents domaines participant au processus, que ce soit du côté de l'offre ou de la demande. Ces deux aspects sont développés ici de façon successive, en prêtant attention à l'interaction des institutions et des contrats, ainsi qu'à ses conséquences en matière de performance des marchés de défense.

96 Economie et Institutions - n²12\&13-2008-2009 


\subsection{Du côté de l'offre : lien maître d'œuvre industriel - sous-traitants}

La valeur élevée des contrats d'armement, s'élevant dans notre échantillon à 135 millions d'euros, écarte de fait les petites et moyennes entreprises de ces marchés. Ces derniers sont ainsi obtenus, pour leur grande majorité, par des grandes entreprises de l'aéronautique, de l'électronique et/ou des secteurs terrestre et naval telles que Thales, EADS, Dassault, Safran ou encore Nexter et DCNS.

Ces dernières jouent un rôle de maître d'œuvre industriel dans la mesure où elles ont la responsabilité, partielle ou totale, des actions nécessaires à l'approvisionnement de systèmes de défense pour le compte de la Délégation générale pour l'armement, qui assure de son côté la maitrise d'ouvrage. Les grandes entreprises de Défense sont devenues dans le temps des intégrateurs à travers le recours de plus en plus souvent, et de façon significative, à des soustraitants.

Comme indiqué dans le tableau 1, ce recours est cependant associé à un niveau relativement élevé de défaillance dans la mesure où les sous-traitants s'avèrent ex post dans l'impossibilité d'atteindre les objectifs visés pour des raisons techniques (risque $n^{\circ} 6$ ) ou organisationnelles (risque $n^{\circ} 8$ ).

Bien que ce degré élevé de défaillance s'explique en partie par les exigences pointues en matière tant technique qu'organisationnelle provenant des maîtres d'œuvre industriel, en réponse aux objectifs qu'ils ont acceptés dans leur relation avec la DGA, se pose la question de leur sélection. Des facteurs à la fois institutionnels et contractuels interagissent dans cette perspective.

Les sous-traitants sont en premier lieu retenus pas les industriels titulaires du marché DGA de façon libre. Des considérations industrielles et contractuelles priment dans cette perspective. En fonction des partenariats industriels mis en place et les prises de participation dans les entreprises, les maitres d'œuvre industriels sélectionnent leurs sous-traitants.

Ces derniers sont en second lieu soumis à une contrainte supplémentaire dans le cadre d'un éventuel plan d'acquisition encadré par la DGA. Depuis l'an 2000, en cas de marché négocié sans mise en concurrence préalable, le titulaire du contrat doit en effet présenter un plan d'acquisition à l'acheteur public. Le plan 
d'acquisition est un engagement contractuel ${ }^{3}$ dans lequel le maître d'œuvre précise les sous-traitants avec lesquels il a prévu de collaborer. L'objectif de ce plan est triple pour la DGA : s'assurer que les sous-traitants les moins coûteux soient retenus afin de minimiser son coût d'acquisition, que les sous-traitants soient traités avec équité, en élargissant notamment la cible des sous-traitants potentiels, et enfin, promouvoir l'innovation technologique et l'émulation technique dans le cas des études amont. Il s'agit là d'une mesure de politique industrielle.

Bien que le plan d'acquisition présente certains avantages, deux difficultés sont néanmoins rencontrées durant leur mise en œuvre. La première, relevée par Beaufils et al. (2004) p.51, repose sur la contradiction entre la responsabilisation du titulaire et l'ingérence potentielle de la DGA dans le choix des sous-traitants. La DGA vérifie cependant dans les faits uniquement la bonne application de la procédure par le titulaire ${ }^{4}$, mais n'intervient normalement pas dans le choix proposé par le titulaire. En effet, une circulaire stipule dès 1976 que " l'examen en vue de l'acceptation des conditions de paiement ne doit pas conduire la personne responsable du marché à s'immiscer dans les relations du titulaire avec le soustraitant " (circulaire du 7 octobre 1976, modifiée par la circulaire du 31 janvier 1983, relative à la réforme du régime de la sous-traitance dans les marchés publics). Cette circulaire s'applique notamment au cas du plan d'acquisition. Si l'ingérence est cependant effective, alors se pose une seconde difficulté. Il s'agit de l'opposition entre l'objectif de la DGA (obtenir un prix le plus faible possible, en concordance avec le principe de concurrence) et celui du titulaire (satisfaire la DGA en lui proposant un système qui corresponde bien à ses attentes, ce qui le conduit à retenir le sous-traitant qui sera capable de réaliser la tâche demandée, et non pas obligatoirement le moins coûteux). Cet antagonisme conduirait alors à un rejet par la DGA du plan d'acquisition, ce qui entraînerait des retards dans l'exécution du programme, donc des surcoûts. L'idée même de plan d'acquisition et les conditions d'exécution de ce plan font actuellement débat au sein de la DGA.

3 La conséquence du caractère contractuel du plan d'acquisition est qu'en cas de non-respect de ce plan par le titulaire, celui-ci peut voir sa responsabilité pour faute engagée. La résiliation du marché peut alors être prononcée par les juridictions administratives.

${ }^{4}$ La DGA demande aux maîtres d'œuvre industriels de déclarer à l'avance la répartition de leur règle de sélection de leurs sous-traitants : 60/40 ou 40/60 par exemple pour les facteurs technique/financier. Le maître d'œuvre sélectionne ensuite ses sous-traitants. Le rôle de la DGA est alors simplement de vérifier la stricte application des critères de sélection énoncés précédemment. Un tel contrôle n'est cependant pas toujours simple à mener.

98 Economie et Institutions $-\mathrm{n}^{\circ} 12 \& 13-2008-2009$ 
Les institutions appellent ainsi, à travers la mise en œuvre du plan d'acquisition, à une diversification et des critères et des responsabilités dans le choix de la sous-traitance. Elles ne se limitent donc pas à l'exécution des accords conclus par les parties dans la mesure où elles sont influentes dans la structuration du contexte de la contractualisation et de la rédaction des accords contractuels, en amont de ces derniers.

\subsection{Du côté de la demande : le lien entre programmes d'armement et marchés publics}

Les marchés d'armement sont, pour l'essentiel, passés dans le cadre de la réalisation des programmes d'armement, dont l'organe exécutif est la DGA. Le processus décisionnel public en matière d'équipements de défense est assez complexe, au regard de la nécessité de définir des besoins opérationnels répondant aux contextes des intérêts de défense et de sécurité, de considérations géopolitiques, de la politique extérieure du pays et d'autres facteurs exogènes. Les Etats-majors, l'Etat major des Armées, le ministère de la défense sont des lieux de discussion et d'arbitrage des programmes d'armement et du cadre de leur réalisation, soit en interne, soit en coopération internationale. Les besoins opérationnels étant définis, leur concrétisation en programmes est confiée à la DGA qui négocie avec les industriels les marchés, planifie leur enchaînement dans le temps, qu'il s'agisse de contrats dits d'études amont visant à "lever les risques" ou de contrats de réalisation portant sur des sous-systèmes, ou sur leur intégration dans des systèmes d'armes. Sans entrer plus avant dans le détail, il importe de souligner que l'articulation des contrats avec l'industrie concernant les programmes d'armement pose trois séries de problèmes.

Le premier est celui de la programmation dans le temps des enchaînements de marchés publics, dans la mesure où ces derniers doivent être passés dans une séquence à temporalité longue. Or, le séquençage des contrats est fréquemment obéré par des risques notamment techniques et contractuels dont la survenance a pour effet de créer des retards qui se cumulent et mettent en péril la programmation budgétaire initiale. Le risque de défaut des engagements budgétaires est alors réel, d'autant plus que les lois de programmation militaires ne sont pas réellement contraignantes et que le ministère du budget arbitre souvent entre affectations des ressources publiques dans le cadre de mesures de "régulation budgétaire" qui se traduisent par des gels, annulations ou reports de 
crédits de paiement qui font peser de lourdes contraintes sur le déroulement des programmes (risques $n^{\circ} 9$ et 10 du tableau 1 ).

Le deuxième découle du fait que l'appareil de suivi financier de l'équipement des forces armées est avant tout budgétaire. La France se singularise par le fait que son appareil administratif se situe essentiellement au niveau de l'analyse (ex ante) des phases d'appel d'offre et de contractualisation pour les programmes d'armement, et n'est pas adapté à l'investigation de l'économie des contrats après leur réalisation.La nomenclature budgétaire ne permet pas, naturellement, d'assurer un suivi de la dépense au niveau des marchés (Kirat et Bayon, 2006). Il convient cependant de souligner que la mise en place progressive, qui sera effective dès 2010, d'un nouveau système financier interministériel (Chorus) vise notamment à répondre à ces difficultés de suivi budgétaire (Lory, 2008).

Le troisième est lié au fait que des retards accumulés au cours de programmes d'armement longs induisent souvent l'obsolescence de sous-systèmes déjà livrés, qui induisent des coûts de transaction non négligeables $d u$ fait de la conduite de négociations entre la DGA et le maître d'oeuvre industriel sur la prise en charge du coûts du "traitement des obsolescences".

Les conditions dans lesquelles les marchés de défense liés aux programmes d'armement montrent que, dans ce domaine, les contrats ne sont pas auto exécutoires, mais s'inscrivent dans un système multi-niveaux à gouvernance complexe, où interviennent des autorités militaires (états-majors), des autorités budgétaires (contrôleur financier au sein de la Défense et direction du budget au ministère des finances), des organes opérationnels (DGA) et de soutien (Secrétariat général pour l'administration) voire des autorités politiques (cabinet du ministre et ministre en charge de la défense). Parmi ces autorités et institutions dont les fonctions de préférence sont hétérogènes, des arbitrages à base de rapports de force et d'influence sont fréquemment menés, dont certains ont été documentés dans la littérature (Kirat, Bayon, Blanc, 2003) : d'une part, les dépassements de prévisions budgétaires dans les programmes d'armement conduit à en sacrifier certains pour poursuivre l'abondement d'autres, sans que ces choix soient forcément cohérents avec les besoins opérationnels des forces armées ; d'autre part, dans les marchés d'équipement passés selon la doctrine de la DGA (contrats pluriannuels, forfaitaires, à rémunération en partie fondée sur des indicateurs de performance), l'incertitude sur la disponibilité en temps et en heure des crédits de paiement (risques $n^{\circ} 9$ et 10 tu tableau 1) conduit les services

100 Economie et Institutions $-\mathrm{n}^{\circ} 12 \& 13-2008-2009$ 
acheteurs à faire preuve d'une grande ingéniosité pour gérer ce risque compte tenu de ses incidences sur les intérêts moratoires de retard légalement dûs aux cocontractrants de l'Etat. La mise en place en 2009 du Comité ministériel d'investissement vise notamment à répondre à ces difficultés d'arbitrage. Il est cependant encore trop tôt pour apprécier ses conséquences en matière d'exécution des marchés.

\section{Section 3 - La renégociation des contrats}

Dans l'approvisionnement de défense en France, la quasitotalité des contrats sont à prix ferme (Kirat, Bayon et Blanc, 2003 ; Oudot, 2007). Ce choix du prix ferme peut s'expliquer par des facteurs institutionnels: aucune loi ne contraint en effet les titulaires des contrats à révéler leurs informations sur les coûts effectifs de réalisation et, surtout, l'efficience des contrôles de coûts est relative, même si des efforts sont actuellement menés dans cette perspective (Beaufils et al., 2004). Dans les années 1980, les faiblesses des contrôles de coûts associées à la révélation d'excès de la part de certains titulaires des contrats sur les coûts déclarés de consommations intermédiaires notamment, ont conduit les autorités publiques à abandonner les contrats à remboursement de coûts dans l'approvisionnement de défense en France. Il est ainsi rappelé que le choix du type de contrat est contraint par l'environnement institutionnel, qui rend les contrats à remboursement de coûts $a$ priori inefficaces dans ce secteur, ce qui doit être nuancé à la marge selon les situations propres à la France et aux Etats-Unis.

Ces prix fermes sont cependant renégociés dans notre base de données dans $56 \%$ des cas, ce qui constitue un changement fréquent des conditions d'exécution des marchés. Un contrat donne lieu en moyenne à deux avenants. La distribution du nombre d'avenants par contrat est présentée dans le graphique suivant :

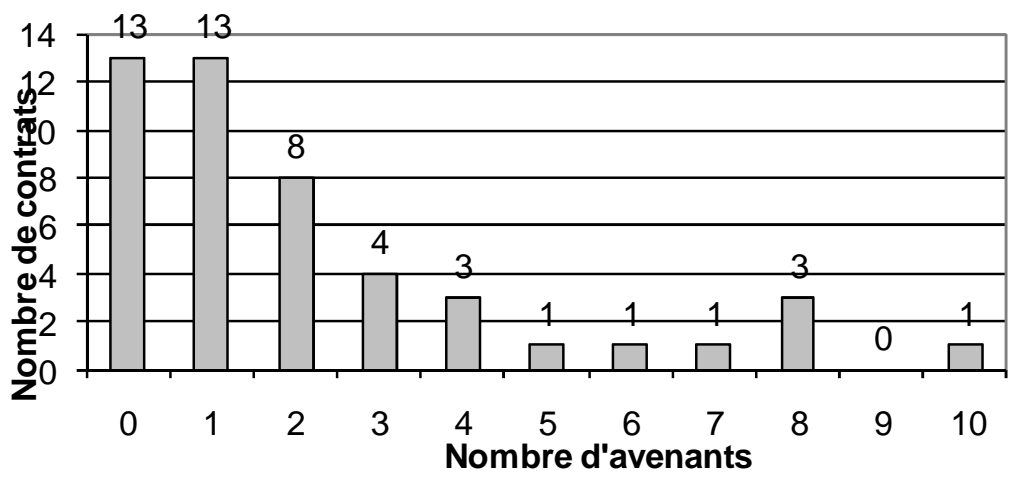

Graphique 1 : nombre d'avenants par contrat

101 Economie et Institutions - n²12\&13-2008-2009 
Ce graphique indique par exemple que dans 13 contrats, aucun avenant n'a été signé, alors qu'un contrat a fait l'objet de 10 avenants. La fréquence des renégociations en général et leur importance dans certains marchés d'autre part appelle à s'interroger sur les conditions d'exécution des contrats. Après avoir étudié leurs déterminants, nous analysons leurs conséquences tant d'un point de vue contractuel qu'institutionnel.

\subsection{Les déterminants des renégociations}

\section{Analyse institutionnelle}

Les concepts d'imprévision, de sujétions imprévues, de force majeure, sont surtout analysés par les juristes du point de vue des principes et des normes juridiques : la doctrine définit des principes généraux sur lesquels les juges administratifs sont censés s'appuyer pour statuer sur des demandes de révision du prix ou de compensation des surcoûts formulées par les cocontractants.

Si l'on passe d'un point de vue doctrinal à une analyse empirique, il s'avère que les travaux disponibles montrent que, en règle générale, les affaires en imprévision se résolvent rarement en faveur des demandeurs. Les conditions exigées pour admettre le rééquilibrage du contrat via une indemnité d'imprévision sont tellement restrictives qu'elles sont dirimantes : l'aléa doit être extérieur aux parties, hors de leur contrôle, être irrésistible et, de plus, doit avoir pour effet de "bouleverser" l'économie du contrat (pour une comparaison FR-USA, Kirat et Vidal 2008) ${ }^{5}$. S'il existe un écart considérable entre le principe et les réalités empiriques dans les marchés publics civils, les marchés de défense sont empiriquement très rarement portés devant le juge administratif. D'autres procédures de régulation que juridictionnelles sont mobilisées, par la renégociation, la passation d'avenants voire par des décisions discrétionnaires prises au plus haut niveau du ministère de la défense.

5 La "Bible" des personnes publiques en la matière, à savoir la circulaire du Premier ministre et du ministre de l'économie des finances du 20 novembre 1974 relative à l'indemnisation des titulaires de marchés publics en cas d'accroissement imprévisible de leurs charges économiques (J.O. du 30 novembre 1974, p. 11971) précise clairement que l'imprévision suppose la réunion de trois éléments: (a) le caractère non raisonnablement prévisible de l'événement perturbateur, (b) le fait que cet événement soit indépendant de la volonté du titulaire et se soit imposé à lui, (c) le fait que l'événement ait occasionné des charges supplémentaires, extracontractuelles, entraînant le bouleversement de l'économie du contrat.

102 Economie et Institutions - n $12 \& 13-2008-2009$ 
En fait, la réglementation des marchés publics de la défense donne, comme dans les autres marchés, des pouvoirs discrétionnaires à l'administration, de l'échelon directement opérationnel qu'est le pouvoir adjudicateur (la personne responsable du marché dans l'ancienne terminologie du code) au ministre en charge de la défense, en passant par les états-majors et les responsables de programmes au sein de la DGA.

L'octroi de pouvoirs décisionnels discrétionnaires ne doit pas être analysé comme le signe d'une défaillance de la réglementation mais, au contraire, comme organisé par elle. Par exemple, la circulaire de 1974 contribue à renforcer la texture ouverte de la théorie de l'imprévision : elle ne proscrit pas l'argument d'imprévision de la part de titulaires de marché à prix ajustable ou révisable et confirme le pouvoir discrétionnaire des autorités contractantes en matière d'appréciation de la justesse des réclamations des titulaires et de suites à leur donner. Dans ces conditions, il est raisonnable de penser que les demandes d'indemnité d'imprévision font l'objet de solutions non contentieuses, et sont de ce fait inobservables: les décisions des personnes responsables et du comité de règlement amiable des litiges dans les marchés ne sont pas publiques, donc pas publiées.

Il n'est pas contestable qu'en matière de défense le recours au juge du contrat n'est pas un procédé courant ou usuel de règlement des différends en cas de sujétions imprévues, d’imprévision ou de force majeure (Simonel, 2008). Ce fait différencie la situation française de celle du plus grand pays producteur d'armements, à savoir les Etats-Unis où le procédé usuel de règlement des différends d'exécution est juridictionnel, devant l'ASBCA (Kirat, Bayon et Blanc, 2003).

Le cadre institutionnel des contrats a donc une influence sur les conditions de leur régulation : à la pratique des avenants en France peut être opposé le recours au juge du contrat aux EtatsUnis. De façon à obtenir une compréhension complète de l'occurrence des renégociations, il est ainsi utile de compléter cette analyse institutionnelle par une étude des conditions d'exécution des contrats.

\section{Analyse contractuelle}

Les renégociations interviennent lorsqu'un décalage entre les conditions effectives d'exécution du marché, en termes de spécification technique, de délais ou de coûts, divergent de celles initialement convenues à la signature du contrat. Des considérations à la fois d'équité et d'efficacité sont prises en compte dans cette

103 Economie et Institutions $-\mathrm{n}^{\circ} 12 \& 13-2008-2009$ 
perspective. Un tel décalage intervient lorsque des risques se matérialisent. Ainsi, $100 \%$ des contrats renégociés dans notre base de données sont caractérisés par l'occurrence d'évènements adverses, tels que présentés dans le tableau 1.

Cette concomitance n'est cependant pas suffisante pour expliquer les renégociations dans l'approvisionnement de défense en France. Il est en effet prévu initialement que l'ensemble des risques soit supporté par le(s) titulaire(s) du contrat. Les clauses de prix sont fermes dans $98 \%$ des cas, les titulaires des marchés sont soumis à une obligation de résultats que ce soit en termes de délais ou de performance technique, pendant que les clauses de rendez-vous, stipulant les conditions sous lesquelles des renégociations peuvent avoir lieu, sont quasiment absentes des contrats d'armement en France.

Alors que des facteurs formels ne peuvent être mobilisés pour justifier les renégociations, celles-ci peuvent être expliquées par des règles non écrites établies entre les parties à l'échange. Ces règles reposent en particulier sur l'allocation des risques à retenir durant l'exécution des marchés, dont les principes reposent sur le principe de responsabilité et le principe d'équilibre des aléas (Oudot, 2007). Les contrats d'armements reposent ainsi en France en partie sur un fonctionnement informel, dans le cadre strict de la réglementation en vigueur.

Ces choix contractuels peuvent en partie être expliqués par des facteurs institutionnels. En parallèle à la complexité des contrats d'armement, il convient de souligner les conséquences de la rationalité limitée des agents (Williamson, 1976) mais aussi des caractéristiques institutionnelles (Laffont, 2005 ; Estache \& WrenLewis, 2009). La position de la DGA dans le processus d'approvisionnement, détaillée dans la section 2.2, les possibilités offertes à la partie publique de modifier ex post de façon unilatérale les conditions d'exécution de tout marché public et la fréquence des transactions entre acteurs dans l'approvisionnement de défense participent à expliquer la nature relationnelle des contrats d'une part et la fréquence de leur renégociation d'autre part.

\subsection{Conséquences des renégociations}

\section{En matière contractuelle}

Les renégociations donnent lieu à une augmentation du prix du contrat, à une validation des retards pris dans le projet et/ou à une réduction de la quantité commandée. Cette dernière a été réduite jusqu'à $30 \%$ dans notre base de données, à périmètre

104 Economie et Institutions - n¹2\&13-2008-2009 
budgétaire constant. Cette solution contractuelle est retenue lorsque la contrainte budgétaire de la DGA (ou de l'État Major des Armées, gouverneur de crédits) est particulièrement tendue. La hausse moyenne des prix dans notre échantillon causée par chaque renégociation s'élève à environ 2 millions d'euros par contrat, dont le prix augmente ainsi en moyenne de près de $5 \%$ du prix initial du marché.

Bien que les conséquences tant calendaires, techniques que financières puissent faire l'objet d'évaluations, il est délicat de les apprécier. L'évaluation de la pertinence des renégociations a en effet donné lieu à des résultats contrastés dans la littérature économique. D'un côté, Jolls (1997) a montré, dans un cadre d'agence, que toute renégociation et toute anticipation de renégociation(s) annihilaient l'efficacité des incitations véhiculées par les termes contractuels. Guasch (2004) insiste lui sur les hausses des prix consécutives aux renégociations ainsi que sur les pertes de bien-être associées à ces hausses de prix. D'un autre côté, Gil (2006) a montré que les renégociations peuvent être perçues comme positives dans la mesure où elles permettent de répondre à la contrainte de participation de l'Agent. Felli \& Villas-Boas (2000) ont par ailleurs démontré que les renégociations peuvent être perçues comme efficaces dans la mesure où elles permettent de réduire les inefficacités causées par une collusion initiale entre les parties à l'échange. Enfin, certains auteurs ont mis en avant à la fois les côtés positifs et négatifs des renégociations. Guasch, Laffont \& Straub $(2006,2007)$ mettent par exemple en exergue leurs effets positifs lorsque l'objectif est de s'adapter aux chocs (non contrôlables) intervenant durant la mise en œuvre du contrat, et les effets négatifs lorsque les parties à l'échange tendent à exploiter de façon opportuniste les faiblesses de l'environnement institutionnel. Baron (1988) établit un résultat similaire. L'analyse de ces travaux, qui s'inscrivent dans des cadres analytiques différents, montre la diversité des résultats des recherches visant à apprécier la pertinence des renégociations. Dans un cadre transactionnel, ces renégociations résultent d'arbitrages entre les coûts de maladaptation en cas de non renégociation et les coûts directs induits par ces renégociations. Dans la mesure où les coûts de maladaptation sont difficilement mesurables, et étant donné la diversité des interprétations possibles, il est ici délicat de conclure sur la pertinence des renégociations intervenues dans l'approvisionnement de défense d'un point de vue contractuel. En revanche, les conséquences institutionnelles sont plus catégoriques.

\section{En matière d'analyse institutionnelle}

On s'intéresse ici au contexte institutionnel des renégociations des contrats, dans la mesure où la vie des contrats

105 Economie et Institutions - n²12\&13-2008-2009 
n'est pas entièrement structurée par les accords entre les parties. L'analyse comparée des mécanismes d'ajustement de l'équilibre financier des contrats passés dans le cadre de la défense montre en effet que les renégociations ne sont pas un mode universel d'adaptation du contrat et que, au sein des renégociations, les marges d'adaptation sont différenciées.

Les renégociations qui se concluent par la passation d'avenants positifs (c'est-à-dire qui se traduisent par une hausse du montant du contrat initial) sont fréquentes en France, alors qu'elles le sont moins dans le cas des Etats-Unis. Le motif de cette différenciation tient dans les caractéristiques institutionnelles de ces deux pays : en France, le recours au juge est rarement pratiqué dans le domaine des marchés de la défense, mais jusqu'à la réforme du Code des marchés publics en 2006, la passation d'avenants positifs était soumise à un avis consultatif obligatoire de l'ex Commission spécialisée des marchés compétente (AMA). Cette dernière donnait rarement des avis négatifs, et appréciait avec largesse le standard de non-bouleversement de l'économie de la convention initiale, sans s'en tenir à une limite de variation du prix strictement définie (Kirat, Bayon et Blanc, 2003). Mais, ce qui importe avant tout est le fait que, sur la période 1993-1999, le développement du recours à des marchés initiaux à prix ferme est concomitant à la passation d'avenants à prix révisable ${ }^{6}$. En d'autres termes, l'hypothèse d'une "métamorphose" des formes de prix peut être retenue. Dans le cas des Etats-Unis, les contrats donnent lieu plus régulièrement à des recours devant le juge compétent, qui opère comme un régulateur central de l'équilibre du contrat et prohibe, le plus souvent, les changements dans la formule de prix initiale.

Outre les renégociations des formes de prix, celles qui touchent des contrats forfaitaires posent également le problème des performances de l'environnement institutionnel : en théorie, un contrat à forfait doit faire assumer au cocontractant de l'Etat le risque de marges inférieures à la marge attendue, voire de profits négatifs. En la matière, la politique de contractualisation de la DGA qui consiste à recourir à des procédures négociées non concurrentielles et à des contrats forfaitaires pluriannuels présente un bilan contrasté.

En théorie, si le contrat à prix forfaitaire a été attribué au terme d'une procédure concurrentielle plutôt que négociée, le cocontractant peut être exposé à la malédiction du vainqueur (Naegelen, 1986) de l'adjudication, dans la mesure où la stratégie de

6 Cette étude n'a malheureusement pu être actualisée depuis.

106 Economie et Institutions $-\mathrm{n}^{\circ} 12 \& 13-2008-2009$ 
maximisation de l'espérance de l'attribution du contrat passe par la formulation d'une offre excessivement optimiste qui sera mise en cause au stade de l'exécution et de ses conditions économiques. De ce point de vue, les marchés négociés devraient permettre de prévenir ces situations de malédiction du vainqueur. En réalité, il n'en va pas systématiquement ainsi : les sous-estimations du devis initial et des éléments de coûts sont pratiquées dans le cadre d'une concurrence entre responsables de programmes d'armement dans un contexte de budgets contraints, chaque service acheteur s'employant à minimiser les estimations a priori du coût des achats pour maximiser l'espérance d'allocation de ressources budgétaires. Dans ce cadre, l'attribution de contrats dont le prix est sous-estimé ne peut manquer de provoquer des difficultés d'exécution et, ainsi, la survenance de risques, contractuels en particulier, obérant le rythme et les conditions économiques de la réalisation des prestations.

La politique de la DGA de recourir à des contrats pluriannuels, forfaitaires et à rémunération basée sur un/des indicateurs de performance (par exemple la rémunération à l'heure de vol des avions dans les contrats de maintenance) annihile en partie les effets positifs de la négociation qui prémunit théoriquement contre la malédiction du vainqueur des procédures concurrentielles. En effet, les prix forfaitaires peuvent donner lieu à la nécessité de procéder à des ajustements, surtout lorsque le volume initial des commandes est réduit à la baisse de manière discrétionnaire par $l^{\prime} E$ tat (risques $n^{\circ} 9$ et 10 , tableau 1). Dans ce cas de figure, des décisions et contraintes extérieures au contrat en affectent directement les conditions économiques, et font des contrats initiaux à prix forfaitaire des engagements dont la crédibilité est faible.

A cet égard, les contrats forfaitaires présentent d'autres limites. Comme Bajari et Tadelis (2001) l'ont montré à partir d'une analyse empirique de contrats de travaux publics en Californie, les deux formes de prix que sont les contrats de type " cost plus " et les prix forfaitaires ("fixed price") ne sont pas exposées de la même manière aux risques de renégociation. Les contrats à prix forfaitaires véhiculent des coûts d'adaptation ex post qui peuvent être assimilés à des coûts de transaction qui réduisent les effets incitatifs recherchés ex ante à destination du contractant. Dans le cas des marchés de la défense, néanmoins, rares sont les procédures d'attribution concurrentielle des contrats, au regard des spécificités des transactions (considérations de secret, de maintien d'une base industrielle et technologique de défense, de sécurité d'approvisionnement, sans omettre les prestations induites par la nature du stock d'équipements de défense dans les forces armées). Dans notre base de données, moins de $25 \%$ des contrats ont ainsi

107 Economie et Institutions - n² $12 \& 13-2008-2009$ 
été passés en suivant une procédure concurrentielle. Le recours à des procédures négociées, avec ou sans publication préalable, est la procédure dominante dans ce domaine. L'intérêt premier de la procédure négociée est de permettre la discussion sur les éléments de coûts proposés par l'industriel, toutefois dans les limites des données de coûts dont l'autorité publique dispose et de ses capacités réglementaires d'enquêtes sur les coûts dans l'industrie. De plus, la négociation ne prémunit pas, en elle-même, du risque de couverture des risques par l'industrie sous la forme de provisions contractuelles, c'est-à-dire par la perception d'une prime de risque dont la probabilité est d'autant plus grande que le contrat est à prix forfaitaire (Oudot, 2008b).

\section{Conclusion}

La performance des marchés passés pour les besoins de la défense est une question qui appelle la prise en compte des dispositions contractuelles bilatérales d'une part, de l'environnement institutionnel d'autre part et, surtout, de leurs interactions. Les éléments empiriques avancés dans cet article confortent cette idée, qui peut être mise en rapport, dans un cadre comparatif, avec la diversité internationale des systèmes de réglementation des acquisitions d'équipements de défense (Kirat, 2008).

L'interaction des facteurs contractuels et des facteurs institutionnels dans les marchés de la défense a été située à deux niveaux : celui des interdépendances de facto entre marchés, soit liés à la sous-traitance du maître d'oeuvre industriel, soit liés aux phases successives des programmes d'armement. Un autre niveau d'interaction entre le contrat et l'environnement institutionnel concerne les renégociations des contrats, pour lesquelles l'évidence empirique montre la fréquence, et à l'occasion desquelles des contradictions entre les effets attendus de pratiques de contractualisation négociée à prix forfaire et les effets du cadre institutionnel dans lequel ils se situent.

\section{Références}

Arrun $\square$ ada B., 2001, "The role of institutions in the contractual process", in T. Kirat, B. Deffains (Eds), Law and Economics in Civil Law Countries, JAI Press, The Economics of Legal Relationships Series, p. 149-167.

Bajari P. \& Tadelis S., 2001; "Incentives versus Transaction Costs: A Theory of Procurement Contracts", Rand Journal of Economics, 32(3), 387-407. 
Baker G., Gibbons R. \& Murphy K.J., 2002; "Relational Contracts and the Theory of the Firm", The Quarterly Journal of Economics, 117, 39-83.

Baron D.P., 1988; "Procurement Contracting: Efficiency, Renegotiation and Performance Evaluation", Information Economics and Policy, 3(2), 109-142; reprinted in Hartley K. \& Sandler T. (Eds.), 2001; The Economics of Defence, Edward Elgar, Cheltenham (U.K.).

Bazzoli L., Kirat T., 2003, "A propos du réalisme en économie des institutions et ses implications sur l'analyse des fondements juridiques des transactions économiques : Commons versus Williamson", Economie appliquée, Tome LVI - $n^{\circ} 3$, sept. 2003, pp. 171-210.

Beaufils R., Bergeal C., Breville A., Chardigny C., Deloison I., Kurz N., Lazar R., Lignières P., Rouilloux J., Warufsel B., 2004; "Les marchés publics de la défense nationale", Contrats publics: l'actualité de la commande et des contrats publics, 32, avril, 39-65.

Beuve J. et Saussier S., 2008; "Enhancing Cooperation in Interfirm Relationships: The Role of Reputation and (In)Formal Agreements", SSRN Working Paper.

Brousseau E. et Raynaud E., 2007; "The Economics of Multilevel Governance", Working paper.

Chakravarty S. \& MacLeod B., 2004; "On the Efficiency of Standard Contracts: the Case of Construction", Working Papers 874, Princeton University.

Deakin S., Wilkinson F., 2000, "Coopération, droit des contrats et performances économiques, in T. Kirat, E. Serverin (dir.), Le droit dans l'action économique, CNRS Editions, p. 159-177.

Estache A. \& Wren-Lewis L., 2009, "Towards a Theory of Regulation for Developing Countries: Following Jean-Jacques Laffont's Lead", Journal of Economic Literature, à paraitre.

Felli L. \& Villas-Boas J.M., 2000; "Renegotiation and Collusion in Organizations", Journal of Economics \& Management Strategy, 9(4), 453-483.

Gil R., 2006; "Renegotiation, Learning and Relational Contracts", University of California Santa Cruz working paper.

Guasch J.L., 2004; Granting and Renegotiating Infrastructure Concessions. Doing it Right. World Bank Institute development studies, Washington.

Guasch J.L., Laffont J-J. \& Straub S., 2006; "Renegotiation of Concession Contracts: A Theoretical Approach", Review of Industrial Organization, 29(1-2).

Guasch J.L., Laffont J-J. \& Straub S., 2007; "Concessions of Infrastructure in Latin America: Government-led

109 Economie et Institutions - n ${ }^{\circ} 12 \& 13-2008-2009$ 
Renegotiations", Journal of Applied Econometrics, 22(7), 12671294.

Jolls C., 1997; "Contracts as Bilateral Commitments: A New Perspective on Contract Modification", Journal of Legal Studies, 26, 203-237.

Kirat T., 2008, "Réglementation et performances", Défense nationale et sécurité collective, $\mathrm{n}^{\circ}$ spécial "Les marchés publics de la défense" (sous la direction de J.-M. Oudot), hors série $\mathrm{n}^{\circ} 12$, 65-74.

Kirat T., Bayon D. \& Blanc H., 2003 ; Maîtriser les coûts des programmes d'armement. Une analyse comparative de la réglementation des marchés industriels d'armement en France, au Royaume-Uni et aux États-Unis, La documentation française, Paris.

Kirat T., Bayon D., 2006, Les marchés publics de la défense. Droit des marchés publics, pratique administrative et enjeux économiques. Editions Bruylant, Bruxelles.

Kirat T. et Vidal L., 2008, "Litigation on Public Contract Performance: A Comparative Study of the Treatment of Additional Costs and Contract Equilibrium by Administrative Judge in the United States and France", Public Contract Law Journal, 381, Fall, p. 153-185.

Laffont J-C., 2005; Regulation and Development, Cambridge: Cambridge University Press.

Lory R., 2008; "Le suivi budgétaire, financier et comptable des marchés publics de défense ", Défense Nationale et Sécurité Collective, $\mathrm{n}^{\circ}$ spécial sur les marchés publics de défense, 5156.

Naegelen F., 1986, "La malédiction du vainqueur dans les procédures d'appel d'offres", Revue économique, $n^{\circ}$ 4, juillet, 608-636.

Oudot J-M., 2007 ; "Choix contractuels et performances : le cas des contrats d'approvisionnement de défense ", thèse de doctorat en sciences économiques, Université Paris I Panthéon Sorbonne.

Oudot J-M., 2008a; "Risques et performance des contrats d'armement ", Défense Nationale et Sécurité Collective, février, $\mathrm{n}^{\circ} 2008-2,132-140$.

Oudot J-M., 2008b; "Choix du type de contrat et performance : le cas des marchés publics de défense ", Economie Publique, 21(2), 157-182.

Pfister E., Defains B., Doriat-Duban M. and Saussier S., 2006; "How laws and institutions may affect organizational choices: The case of franchising", European Journal of Law and Economics, 21(1), 53-78.

Simonel L-X., 2008; "Pour une refondation du règlement du différend né du marché public de défense", Défense Nationale et

110 Economie et Institutions - n¹2\&13-2008-2009 
Sécurité Collective, $\mathrm{n}^{\circ}$ spécial sur les marchés publics de défense, 195-208.

Williamson O.E, 1976; "Franchise bidding for natural monopolies: In general and with respect to CATV", Bell Journal of Economics, 7(1), 73-104.

Williamson O.E, 1985; The Economic Institutions of Capitalism. Firms, Markets, Relational Contracting, The Free Press, New-York.

Williamson O.E, 1996; The Mechanisms of Governance, Oxford University Press, Oxford.

Williamson O.E, 2000; "The New Institutional Economics: Taking Stock, Looking Ahead", Journal of Economic Literature, 38(3), 595-613.

111 Economie et Institutions - n²12\&13-2008-2009 